\title{
Drag Reduction Characteristics of Bionic Structure Composed of Grooves and Mucous Membrane Acting on Turbulent Boundary Layer
}

\author{
K. Zhang ${ }^{1,2}$, C. $\mathrm{Ma}^{1,2}$, J. Zhang ${ }^{3 \dagger}$, B. Zhang ${ }^{1,2}$ and B. Zhao ${ }^{1,2}$ \\ ${ }^{1}$ Department of Mechanical Engineering, College of Engineering, Ocean University of China, Qingdao \\ 266100, China \\ ${ }^{2}$ Key Laboratory of Ocean Engineering of Shandong Province, Ocean University of China, Qingdao 266100, \\ China \\ ${ }^{3}$ Department of Chemistry, College of Chemistry and Chemical Engineering, Ocean University of China, \\ Qingdao 266100, China
}

†Corresponding Author Email: dmh217@ouc.edu.cn

(Received April 19, 2021; accepted SSeptember 9, 2021)

\begin{abstract}
The biological surface structure comprising fish scales and a mucous membrane exhibits good turbulent drag reduction ability. Based on this structure, a bionic frictional drag reduction model composed of a grooved structure and mucous membrane was established herein, and its efficacy in reducing the resistance of a turbulent boundary layer was analyzed. Accordingly, the drag reduction performance of the bionic structure was investigated through large eddy simulations. The results revealed that the mucous membrane was evenly distributed on the groove wall through secretion, and effectively improved the drag reduction rate of the groove wall. The bionic grooves and mucous membrane structure successfully inhibited the turbulent kinetic energy, turbulence intensity, and Reynolds stress. The grooved structure improved the shape of the $\Lambda$ vortex structure and the mucous membrane reduced the number of three-dimensional (3D) vortex structures. Furthermore, the streak structure near the bionic structure wall was reduced and its shape was regularized, which intuitively demonstrates the turbulence suppression ability of the proposed bionic structure. This paper presents the results of a hydrodynamic analysis of the frictional drag reduction characteristics of a bionic structure consisting of grooves and viscous membranes acting on the turbulent boundary layer of a wall.
\end{abstract}

Keywords: Mucous membrane; Bionic drag reduction; Viscoelastic fluid; Turbulence statistics; Coherent structure.

\section{NOMENCLATURE}

$\begin{array}{ll}C_{f s} & \text { drag coefficient of the smooth surface } \\ C_{f m} & \text { drag coefficient of the mucous membrane } \\ D R & \text { drag reduction rate } \\ H & \text { characteristic length } \\ I & \text { turbulence intensity } \\ \operatorname{Re}_{U} & \text { Reynolds number based on } U \\ \operatorname{Re}_{\tau} & \text { Reynolds number based on } u_{\tau} \\ U & \text { flow velocity } \\ U_{i n j} & \text { mucus injection velocity } \\ u^{\prime} & \text { flow direction pulsation velocity } \\ u_{\tau} & \text { wall friction velocity } \\ v^{\prime} & \text { normal direction pulsation velocity } \\ X & \text { flow direction }\end{array}$

$\begin{array}{ll}Y & \text { normal direction } \\ Z & \text { span direction } \\ \gamma & \text { shear rate } \\ \lambda & \text { relaxation time } \\ \mu & \text { viscosity of mucus } \\ \mu_{0} & \text { zero shear viscosity } \\ \mu_{\infty} & \text { infinite shear viscosity } \\ \rho & \text { density } \\ \rho_{m} & \text { density of mucus } \\ \tau_{w} & \text { shear stress } \\ v & \text { kinematic viscosity } \\ <> & \text { time average of a variable } \\ ()^{+} & \text {Non-dimensionalization of a variable }\end{array}$




\section{INTRODUCTION}

The skin-scale structure of fish exhibits excellent turbulent drag reduction performance. Based on this biological structure, several bionic non-smooth surface drag reduction methods have been developed (Zhang et al. 2020a; Liu et al. 2021; Jiang et al. 2016; Cui and Fu 2012; Song et al. 2011; Heidarian et al. 2018). However, the drag reduction effect of these bionic non-smooth surface structures is generally less than $10 \%$. In extreme cases, if the surface structure is improperly arranged or shaped, the drag reduction effect can even be zero. The presence of mucus (viscoelastic fluid) on the scale surface of fish also provides a strong drag reduction effect, and a maximum drag reduction rate $(D R)$ of $80 \%$ can be achieved, as demonstrated by the dragreducing agents (DRAs) used in the field of pipeline transportation. However, this method is useless in working conditions wherein the DRA cannot be injected into the solution. Fish have a drag reduction system composed of a non-smooth surface and a mucous membrane, which supports its efficient swimming in water (Seo et al. 2020; Dou et al. 2012). This system appears to be a promising solution for reducing frictional drag in various applications, especially those in marine environments. Therefore, the frictional drag reduction characteristics of a bionic structure composed of a mucous membrane and grooves must be explored in detail.

A bionic non-smooth surface consists of a series of regular- or irregularly-shaped microstructures that are distributed on a wall based on the Reynolds number length. The most commonly used nonsmooth surface structural shapes are V-shaped, Ushaped, and rectangular grooves distributed along the span or flow direction, or different types of pits, convex structures, and irregular peaks and valleys. Walsh et al. (Walsh 1990; Walsh 1983; Hefner et al. 1983) revealed that a grooved structure has a good drag reduction effect when the height, $s$, and spacing, $h$, of V-shaped grooves have dimensionless size of approximately $s^{+}<25$ and $h^{+}<30$. This theory can also be applied to studies on the drag reduction effect of other non-smooth shapes. The biomimetic non-smooth surface drag reduction method has been widely used primarily owing to its continuity, with almost no losses after processing. Notably, it is also supported by the "second vortex group theory" (Gallagher and Thomas 1984) and the "protruding height theory" (Walsh 1990; Ke et al. 2009).

Bionic mucus drag reduction is an active drag reduction method. Fish mucus can be imitated by using a chemically synthesized viscoelastic fluid, which is injected into the flow field as a DRA to form a mixed solution. This solution is primarily used in pipeline transportation to significantly reduce fluid drag (Majd et al. 2016; Vilalta et al. 2016; Graham 2014). The drag reduction method of secreting mucus on the surface of an underwater vehicle to form a mucous membrane was first proposed by Zhang et al. (Zhang et al. 2020a) After Toms theory (Toms 1948) was proposed, viscoelastic fluid drag reduction became a significant area of research. Viscoelastic fluid DRAs primarily include polymer solutions (Zhang et al. 2020a; Shashank and Sreenivas 2020; Rajappan and McKinley 2020; Xi 2019; Zhu and Xi 2019) and surfactants (Liu et al. 2021; Tamano et al. 2018; Wakimoto et al. 2018; Yanuar et al. 2017), whose drag reduction mechanisms are quite different. In the static state, a worm-like micelle structure is formed in the interior of the surfactant DRA solution; however, when there is a fluid shear force, the worm-like micelle structure tends to incline along the streamline direction, thereby reducing drag. The $D R$ of the DRA gradually increases as the flow Reynolds number (Re) increases, but eventually decreases when the Re reaches a critical value (Cai 2016). The long molecular chain structure of the polymer DRA dissolves in the solvent, and the viscosity of the mixed solution can be significantly improved by increasing the tensile viscosity of the mixed solution. If the viscoelastic relaxation timescale of the fluid is close to the timescale of the turbulent fluctuations near the wall, the regeneration cycle of the near-wall turbulent flow is disrupted resulting in momentum transport perpendicular to the wall, which reduces frictional drag (Zakin et al. 1998).

These studies have clearly demonstrated that both bionic non-smooth structures and mucus have excellent turbulent frictional drag reduction performance. Most current research on drag reduction methods using viscoelastic fluids only focuses on methods wherein a DRA is mixed with the transported fluid to form a mixed solution. However, this method is only suitable for pipeline fluid transportation. In contrast, few studies have been conducted on the application of bionic mucus drag reduction to underwater vehicles. The combination of a biomimetic non-smooth surface structure and a biomimetic mucus film can significantly improve the $D R$ of underwater vehicles. In this study, hydrodynamic simulations are performed to investigate mucus secretion and mucous membrane formation on a bionic groove wall. Subsequently, a comparative study is conducted on the drag reduction performance of groove walls with and without a mucous membrane. Finally, the drag reduction mechanism is clarified based on the turbulence statistics and a coherent structure analysis.

\section{HYDRODYNAMIC MODELING}

A hydrodynamic simulation model of water flow through a rectangular channel was established as shown in Fig. 1. The midpoint of the bottom wall at the entrance was set as the coordinate origin. The streamwise, normal, and spanwise directions were denoted as $\mathrm{X}, \mathrm{Y}$ and $\mathrm{Z}$ respectively, and the characteristic length $H=0.005 \mathrm{~m}$. A bionic structure composed of grooves and a mucous membrane were adopted in the model to reduce turbulence-induced friction drag. A smooth wall, which is a non-dragreducing surface, was placed at the top of the channel for comparison with the bionic structure 
wall. A bionic structure wall with triangular grooves arranged along the spanwise direction was set at the bottom of the channel, and mucus secretion holes were evenly distributed on the platform above the grooves. Mucus was secreted along the normal direction of the wall to form a mucous membrane on the grooved structure.

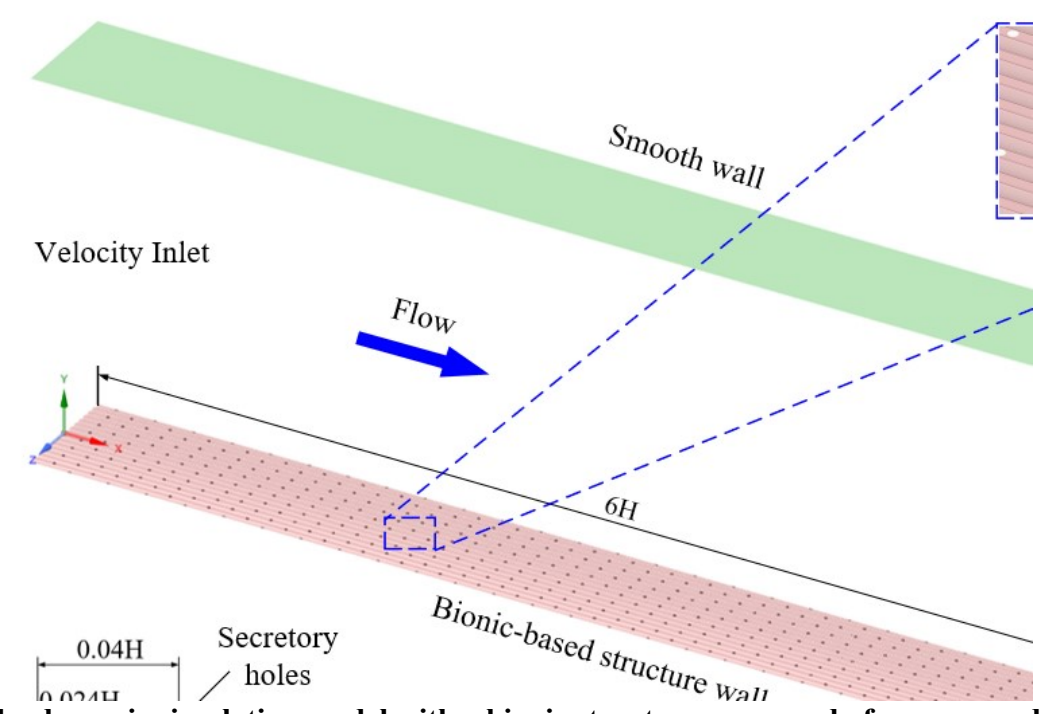

Fig. 1. Hydrodynamic simulation model with a bionic structure composed of grooves and a mucous membrane.

Research has shown that mucus is a non-Newtonian fluid. Several viscoelastic fluid models can simulate the rheological behavior of non-Newtonian fluids, such as Oldroyd-B model (Oldroyd 1950), FENE-P model (Tanner 1975), Giesekus model (Giesekus 1982), etc. Most viscoelastic fluid DRAs are assumed to be viscoelastic fluids. However, it is more appropriate for the constitutive model of fish mucus to be modeled using the Carreau model (Liu et al. 2010). Carreau model fluids behave like Newtonian fluids at low shear rates and like powerlaw fluids at high shear rates, which best represents the rheological properties of fish mucus. The Carreau model can be expressed as Eq. (1).

$$
\frac{\mu-\mu_{\infty}}{\mu_{0}-\mu_{\infty}}=\frac{1}{\left[1+(\lambda \gamma)^{2}\right]^{(1-n) / 2}}
$$

where $\mu_{0}$ is the zero-shear viscosity, $\mu_{\infty}$ is the infinite-shear viscosity, $\lambda$ is the relaxation time, $n$ is the power-law coefficient, $\gamma$ is the shear rate, and $\mu$ is the viscosity of mucus. The specific parameters adopted herein, which are based on the conclusions from previous studies (Liu et al. 2010; Gao 2006; $\mathrm{Xu}$ 2018), are shown in Table $1\left(\rho_{m}\right.$ is the density of mucus).

\section{Table 1 Mucus parameters}

\begin{tabular}{|c|c|c|c|c|}
\hline$\mu_{0} / \mathrm{kg} \cdot \mathrm{m}^{-1} \cdot \mathrm{s}^{-1}$ & $\mu_{\infty} / \mathrm{kg} \cdot \mathrm{m}^{-1} \cdot \mathrm{s}^{-1}$ & $n$ & $\lambda / \mathrm{s}$ & $\rho_{m} / \mathrm{kg} \cdot \mathrm{m}^{-3}$ \\
\hline 0.3 & 0.0005 & 0.3 & 50 & 1000 \\
\hline
\end{tabular}

For numerical calculations, the computational domain was divided using a polygonal unstructured grid. To fully distinguish the turbulent structure near the wall, the normal direction was refined using an unstructured grid, and a boundary layer was established near the wall. The grid height of the first boundary layer was $y^{+}<1$ (nondimensionalized by $u_{\tau}$.). The entrance and the mucus secretion holes adopt a velocity inlet boundary, and the exit adopts an outflow boundary. Owing to the periodicity of turbulence, periodic boundary conditions were used along the Z-axis. The smooth surface and bionic surface were set as frictionless walls.

The transient solver of Ansys Fluent 2019 R3 was used to implement a large eddy simulation (LES) of the proposed hydrodynamic model. Herein, the wall-adapted local eddy (WALE) viscosity model for wall turbulence was selected as the sub-grid stress model. The mixture multiphase flow model was used to simulate mucus secretion in the external flow field, where the mucus can be fused. The pressure-velocity coupled solver and SIMPLEC algorithm were used for calculation. The gradient term was discretized using the Green-Gauss nodebased format, the pressure term was discretized using the PRESTO scheme, and the momentum and volume fractions were discretized using the firstorder upwind scheme. To ensure the accuracy of the calculation, the maximum Courant number of the flow field was set to two. Accordingly, the time step used herein was $0.0003 \mathrm{~s}$, which saves calculation time and resources while ensuring that the transient flow field information is preserved. The velocity at the midpoint of the flow domain changes with time as shown in Fig. 2. The velocities $U=3 \mathrm{~m} / \mathrm{s}$ and $U$ $=5 \mathrm{~m} / \mathrm{s}$ under the two working conditions stabilized after $0.03 \mathrm{~s}$, and the Re values at the end of the flow field were 947.3336 and 1576.049, respectively. The boundary layer was completely turbulent along the wall. The remaining time steps were used for time averaging. 


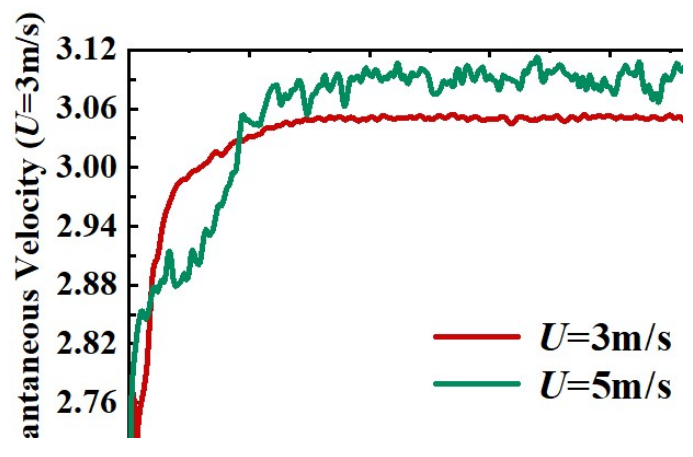

Fig. 2. Relationship between the number of grids and $\boldsymbol{C}_{f .}$.

To verify the accuracy of the model and the calculation method, the grid independence must be verified first. Four sets of grids with minimum wall grid sizes of $0.0001 \mathrm{~m}, 0.00007 \mathrm{~m}, 0.00005 \mathrm{~m}$, and $0.00003 \mathrm{~m}$ were used in the simulation performed herein. The friction coefficients $\left(C_{f}\right)$ of the smooth surface were calculated at $U=3 \mathrm{~m} / \mathrm{s}$ and $U=5 \mathrm{~m} / \mathrm{s}$ using four different calculation models with different grid numbers. The two dashed lines in the bottom of Fig. 3 indicate the theoretical value of $C_{f}$, which was calculated using the Dean formula (Cai 2016) of the turbulent $C_{f}$ in the straight channel.

When the simulation was stable, $C_{f}$ was calculated with 4,236,585 grids (minimum wall grid size $=$ $0.00005 \mathrm{~m}$; growth rate along the normal direction $=1.1$ ) and compared to the theoretical $C_{f}$, as shown in Table 2. As the relative error was within an acceptable range, this number of grids meets the relevant requirements. Therefore, the model with $4,236,585$ grids was used for further calculation, as shown in Fig. 4.

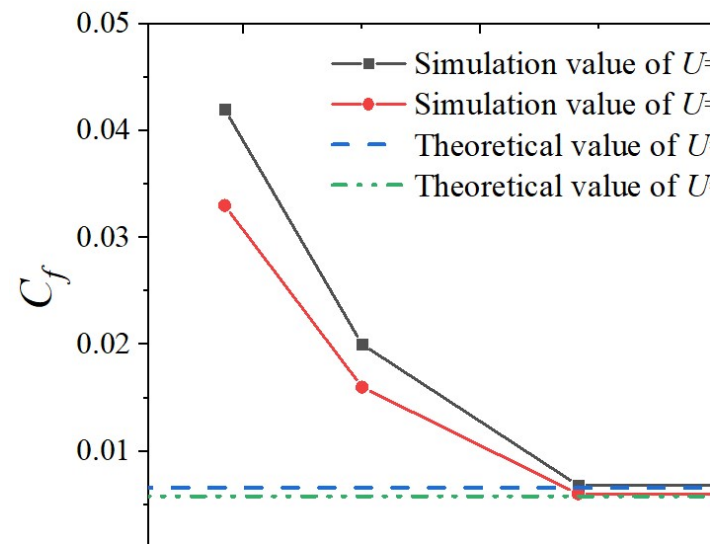

Fig. 3. Relationship between the number of grids and $C_{f}$.

Table 2 Verification of $C_{f}$

\begin{tabular}{|c|c|c|c|}
\hline \multirow{2}{*}{ Condition } & \multicolumn{2}{|c|}{$C_{f}$} & \multirow{2}{*}{$\begin{array}{c}\text { Relative } \\
\text { Error }\end{array}$} \\
\cline { 2 - 3 } & Theoretical & Simulation & \\
\hline$U=3 \mathrm{~m} / \mathrm{s}$ & 0.006596 & 0.006830 & $3.55 \%$ \\
\hline$U=5 \mathrm{~m} / \mathrm{s}$ & 0.005805 & 0.006023 & $3.76 \%$ \\
\hline
\end{tabular}
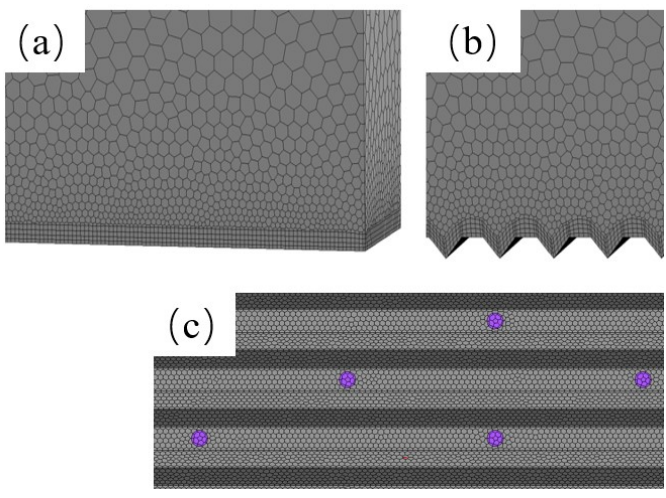

Fig. 4. Computational grid: (a) smooth wall boundary layer; (b) bionic structure wall boundary layer; and (c) mucus secretion holes.

\section{RESULTS AND DISCUSSION}

First, a set of LES simulations were performed to study the secretion of mucus and the formation of the mucous membrane on the bionic groove wall. Subsequently, the drag reduction performance of the groove wall with and without the mucous membrane was studied using the classical turbulent boundary layer theory, and the drag reduction mechanism was analyzed based on the turbulence statistics and coherent structure.

\subsection{Drag reduction effect of bionic structure}

The formation of a mucous membrane is a necessary condition to change the properties of the wall and reduce drag. Figure 5 shows the distribution of the bionic mucous membrane on the wall of the bionic grooves structure for $U=3 \mathrm{~m} / \mathrm{s}$. When $U=5 \mathrm{~m} / \mathrm{s}$, the result was similar to that shown in Fig. 5. Owing to the inlet effect, mucus was sparsely distributed at the front of the flow field, and the secreted mucus was washed to the rear of the flow field by the water. The mucous membrane formed and its thickness gradually increased between $x /(6 H)=0-0.3$; beyond $x /(6 H)=$ 0.3 , the thickness of the mucous membrane became uniform.

The basic parameters of the turbulent boundary layer and the $D R$ under different flow conditions are listed in Table 3. The letters "S", "G", and "GM" in Table 3 indicate that the surface structure was smooth, grooved, and composed of grooves and a mucous membrane, respectively. The numbers "3" and " 5 " indicate flow conditions of $U=3 \mathrm{~m} / \mathrm{s}$ and $U$ $=5 \mathrm{~m} / \mathrm{s}$, respectively. $U_{i n j}$ is the velocity of mucus secretion; $\mathrm{Re}_{\tau}$ is the Reynolds number based on the wall friction velocity $u_{\tau}$, which is defined by Eq. (3); and $C_{f}$ and $D R$ are based on the shear stress $\tau_{w}$ (calculated by the numerical simulation) and are calculated using Eq. (4) and Eq. (5) ( $C_{f A}$ is the friction coefficient with drag reduction and $C_{f B}$ is that without drag reduction), respectively. 


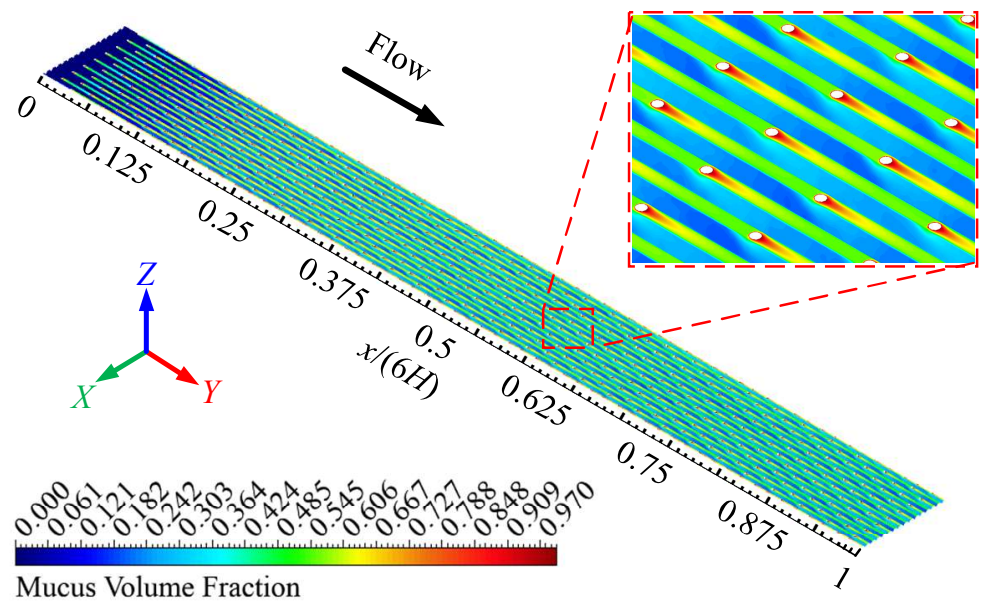

Fig. 5. State of mucous membrane.

Table 3 Basic parameters of turbulent boundary layer and $D R$

\begin{tabular}{|c|c|c|c|c|c|c|c|}
\hline Condition & $U / \mathrm{m} \cdot \mathrm{s}^{-1}$ & $U_{i n j} / \mathrm{m} \cdot \mathrm{s}^{-1}$ & $\mathrm{Re}_{\tau}$ & $u_{\tau} / \mathrm{m} \cdot \mathrm{s}^{-1}$ & $\tau_{w} / \mathrm{kg} \cdot\left(\mathrm{m} \cdot \mathrm{s}^{2}\right)^{-1}$ & $C_{f}$ & $D R$ \\
\hline S-3 & 3 & - & 876.568 & 0.175314 & 30.6795 & 0.006830 & - \\
\hline G-3 & 3 & - & 782.286 & 0.156457 & 24.4348 & 0.005440 & $20.35 \%$ \\
\hline GM-3 & 3 & 0.05 & 772.602 & 0.154520 & 23.8336 & 0.005306 & $22.31 \%$ \\
\hline S-5 & 5 & - & 1371.904 & 0.274381 & 75.1493 & 0.006023 & - \\
\hline G-5 & 5 & - & 1284.780 & 0.256956 & 65.9076 & 0.005282 & $12.30 \%$ \\
\hline GM-5 & 5 & 0.08 & 1226.608 & 0.245322 & 60.0744 & 0.004815 & $20.06 \%$ \\
\hline
\end{tabular}

$$
\begin{gathered}
u_{\tau}=\sqrt{\frac{\tau_{w}}{\rho}} \\
C_{f}=\frac{\tau_{w}}{\frac{1}{2} \rho U^{2}} \\
D R=\frac{C_{f A}-C_{f B}}{C_{f A}} \times 100 \%
\end{gathered}
$$

As shown in Table 3, for the $\mathrm{S}$ structure, $\mathrm{Re}_{\tau}=$ 876.568 at $U=3 \mathrm{~m} / \mathrm{s}$ and $\operatorname{Re}_{\tau}=1371.904$ at $U=5$ $\mathrm{m} / \mathrm{s}$. Under the same conditions, $\operatorname{Re}_{\tau}$ decreased to 782.286 and 1284.780, respectively, for the $G$ structure. This indicates that the $\mathrm{G}$ structure had an inhibitory effect on turbulence. The $\operatorname{Re}_{\tau}$ values of the GM structure were even lower than those of the $\mathrm{G}$ structure, indicating that the former had a stronger inhibitory effect on turbulence. As the drag reduction performance is closely related to turbulence control, the GM structure had a better drag reduction effect than the G structure. Notably, the $G$ structure exhibited an excellent drag reduction effect when $U$ was relatively low (i.e., the turbulence was less chaotic), whereas the mucous membrane in the GM structure had a negligible effect. However, as $U$ increased, the $D R$ of the $G$ structure decreased, and the mucous membrane in the GM structure provided a stronger drag reduction effect.

The average normal dimensionless velocity $\left(U^{+}=\right.$ $\left.U / u_{\tau}\right)$ distribution of the above structures under different flow conditions are shown in Fig. 6 (the streamwise coordinate is $x /(6 H)=0.8$, and the turbulence statistics were obtained using the flow field parameters at this position). For comparison, the well-known Newtonian fluid relationships of the linear law $U^{+}=y^{+}$and the logarithmic law $U^{+}=$ $2.44 \ln y^{+}+5.5$ are illustrated as well. The obtained mean velocity profile of the $\mathrm{S}$ structure surface boundary layer is in good agreement with $U^{+}=y^{+}$ in the viscous sublayer $\left(y^{+}<5\right)$, and $U^{+}=2.44 \ln y^{+}$ +5.5 in the log-law layer $\left(30<y^{+}<0.15 \mathrm{Re}_{\tau}\right)$, further demonstrating the accuracy of the hydrodynamic model. The boundary layers of the $\mathrm{G}$ and GM structures were higher than those of the $S$ structure in the viscous sublayer and log-law layer. The GM structure had the highest average velocity profile, which fully characterizes the drag reduction characteristics of all three structures. Under the shear action of the water flow, the shear-thinning performance of the mucous membrane increases the flow velocity of the boundary layer.

\subsection{Turbulent statistics}

The intensity (I) of turbulence was used to measure the turbulence intensity of the flow field. Figure 7 presents a comprehensive comparison of the $\mathrm{I}^{+}$ 

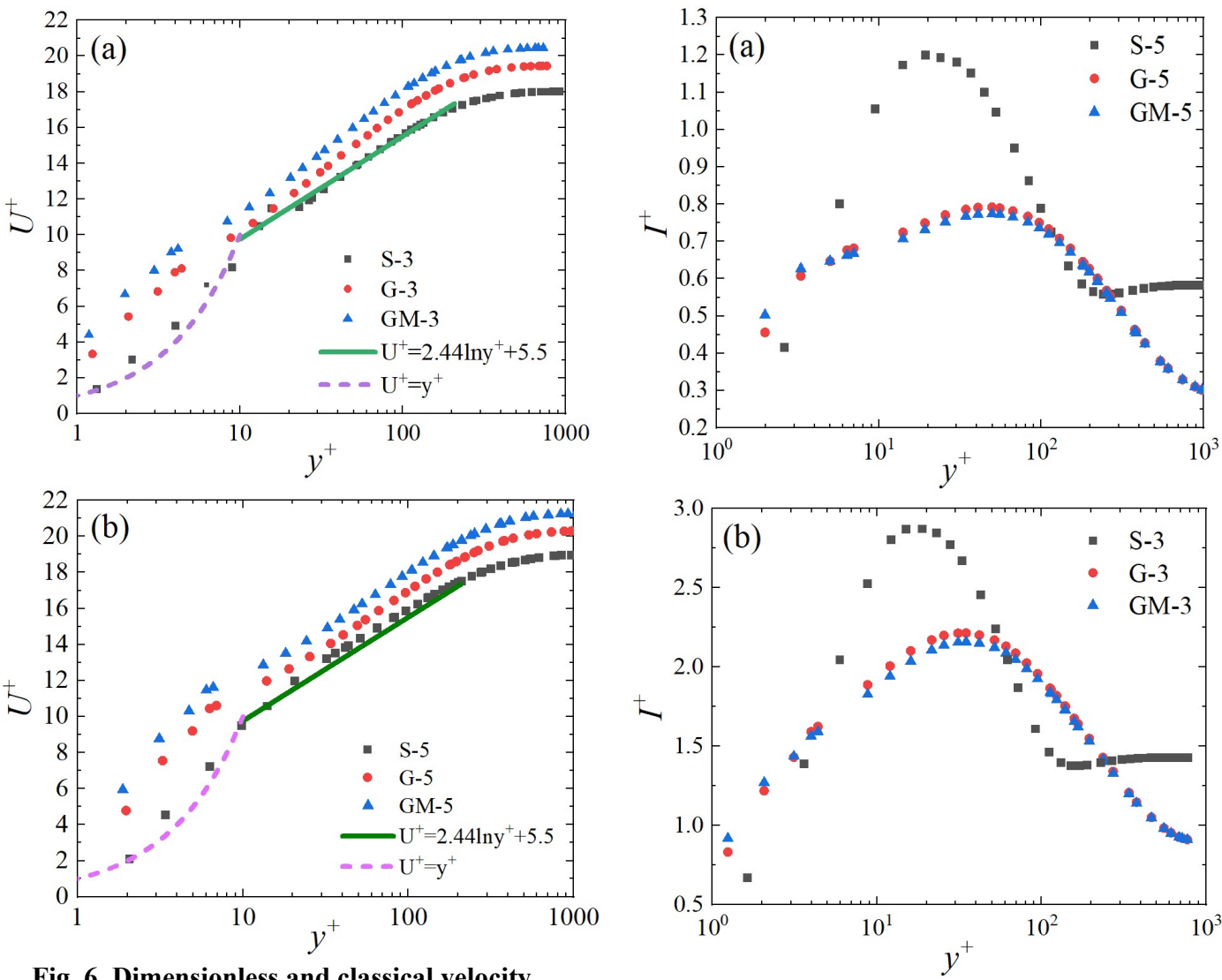

Fig. 6. Dimensionless and classical velocity

profiles: (a) $U=3 \mathrm{~m} / \mathrm{s}$ and (b) $U=5 \mathrm{~m} / \mathrm{s}$.

Fig. 7. Dimensionless turbulence intensity: (a) $U$

$=3 \mathrm{~m} / \mathrm{s}$ and (b) $U=5 \mathrm{~m} / \mathrm{s}$.

values of the S, G, and GM structures under different flow velocity conditions, which were nondimensionalized using $u \tau 2$. The peak values of I+ of the $\mathrm{G}$ and GM structures were significantly lower than that of the S structure, and the peak value of the GM structure was the least. However, the $\mathrm{I}^{+}$distribution of the $\mathrm{G}$ and GM structures was so wide that it covered the entire boundary layer. The peak value of $\mathrm{I}^{+}$of the $\mathrm{S}$ structure appeared near $\mathrm{y}^{+}=20$, and moved outwards $\left(\right.$ near $\mathrm{y}^{+}=50$ ) after drag reduction, thereby reducing the intensity of the turbulence on the near-wall surface. The $\mathrm{I}^{+}$ values of the viscous sublayer on the wall of the $G$ and GM structures were slightly higher than that on the wall of the S structure. This can be attributed to the disturbance of the flow field caused by the grooved structure perpendicular to the wall and the mucus secretion. However, the weak disturbance was limited to the viscous bottom layer and did not significantly weaken the strong drag reduction effects of the $\mathrm{G}$ and GM structures.

In hydrodynamics, turbulence kinetic energy (TKE) is defined as the average kinetic energy per unit mass related to the eddy current in the turbulence. Figure 8 compares the $\mathrm{TKE}^{+}$values of the $\mathrm{S}, \mathrm{G}$, and GM wall structures under different flow velocity conditions, which were nondimensionalized using $u_{\tau}^{2}$. As shown, $\mathrm{TKE}^{+}$reduced after drag reduction, and the peak value of the GM structure was the least. Thus, the GM structure had the strongest effect on reducing the turbulence energy. Furthermore, the $\mathrm{TKE}^{+}$curve of the GM structure

also shifted outward. Therefore, both bionic structures had a stronger effect on $\mathrm{TKE}^{+}$at high Re.

Reynolds stress is defined as the additional stress caused by the momentum exchange generated by the turbulent pulsating velocity. Figure 9 compares the distribution of the Reynolds normal stress $\left(\left\langle u^{\prime} u\right\rangle^{+}\right)$and Reynolds shear stress $\left(-<u^{\prime} v^{\rangle^{+}}\right)$ relative to the normal position of the $\mathrm{S}, \mathrm{G}$, and GM wall structures at different flow velocities. Both stress parameters were nondimensionalized using $u_{\tau}{ }^{2}$. Compared to the $\mathrm{S}$ structure, the value of $\left\langle u^{\prime} u\right\rangle^{+}$on the bionic structure surface reduced significantly, and the peak value also shifted outward. However, there was no significant difference between the values of $\left\langle u^{\prime} u^{\rangle^{+}}\right\rangle^{+}$of the G and GM structures, with the larger value of $\left\langle u^{\prime} u\right\rangle^{+}$ of the GM structure wall in the viscous sublayer. Similarly, the $-\left\langle u^{\prime} v^{\rangle^{+}}\right\rangle^{+}$values shifted outward more significantly. The increase in the stress value of the viscous sublayer of the GM structure wall is primarily related to the fluctuation in mucus secretion.

The above analysis explores the drag reduction performance of the bionic structure wall based on turbulence statistics theory. Both the G and GM bionic structures weakened the statistical peak of turbulence and shifted it away from the wall. This reduced the impact of turbulence on the wall and the frictional drag. The GM structure had a more significant effect on the turbulence statistics. 


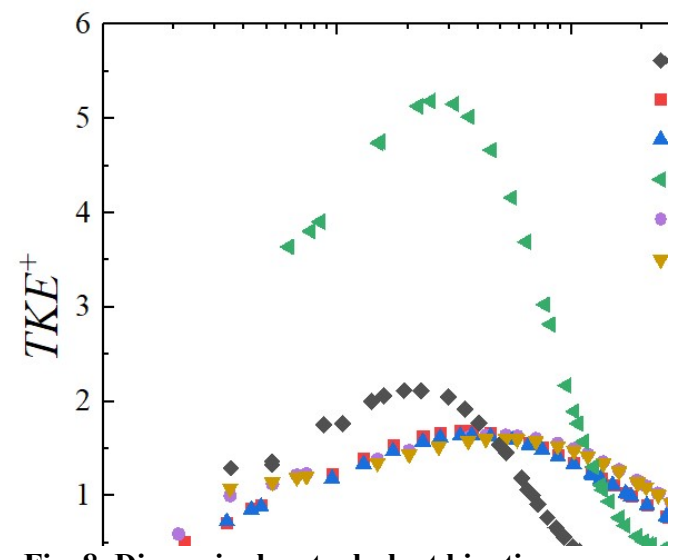

Fig. 8. Dimensionless turbulent kinetic energy.
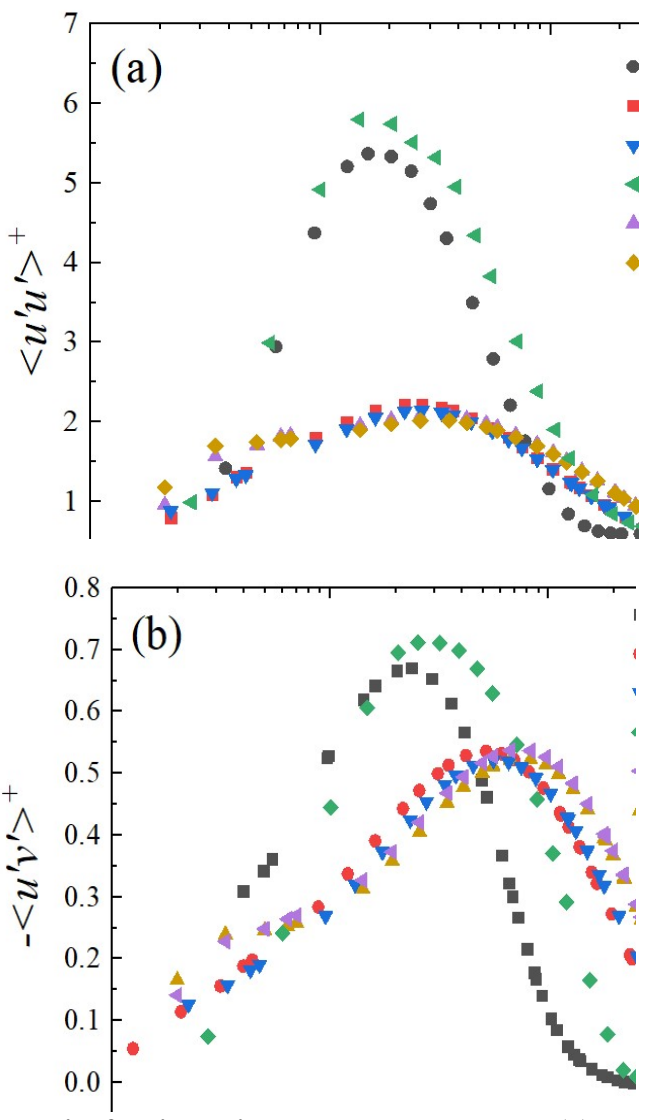

Fig. 9. Dimensionless Reynolds stress: (a) dimensionless Reynolds normal stress and (b) dimensionless Reynolds shear stress.

However, the turbulence statistics cannot clarify the specific structure and details of turbulence. Therefore, the turbulent coherent structure was analyzed to determine the control effect of the bionic structure wall on the former.

\subsection{Coherent structure}

A coherent structure refers to an irregularly triggered orderly movement in shear turbulence. The turbulent pulsation near the wall is constrained by the wall. Studies have shown that the structure of the small-scale vortex near the wall is extremely complex and is the primary source of wall resistance. The basic elements of a coherent structure are the "streak structure" and the "vortex structure" (Zhang 2017), which are located in a fully developed turbulent boundary layer and form a relatively stable circulation mechanism. If any link in this mechanism is destroyed, the self-sustaining process of the turbulent coherent structure can be controlled and turbulent bursts can be suppressed (Asrafi et al. 2019).

Figure 10 and Fig. 11 compare the streak structures (i.e., the instantaneous velocity field) of the boundary layer in the $x-z$ plane at $y^{+}=15$ under different flow conditions. As shown, the velocity streak structure (blue area in the plane) for $U=3$ $\mathrm{m} / \mathrm{s}$ is sparser than that for $U=5 \mathrm{~m} / \mathrm{s}$, which can be attributed to the difference in Re between the two flow conditions. The streak structure of the $\mathrm{S}$ structure was the most chaotic, and the lower speed streaks crossed each other and broke, thereby increasing the wall resistance. The streak structure of the bionic $\mathrm{G}$ structure wall was relatively stable and sparser than that of the $\mathrm{S}$ structure. The wall surface of the bionic GM structure had the best control effect. For $U=3 \mathrm{~m} / \mathrm{s}$, there was almost no streak structure at the end of the plane (i.e., turbulence was stable). Thus, the mucous membrane plays an important role in the formation and evolution of the streak structure.

In a fully developed turbulent boundary layer, the low-speed streaks form a vortex tube under the action of the flow velocity gradient. The vortex tube shifts upward and moves along the spanwise direction. When two streaks collide, they connect to form a $\Lambda$ vortex that is shaped like a capital " $\Lambda$ " and comprises the vortex head with spanwise vorticity, the vortex neck, and the vortex leg with flow vorticity. When the $\Lambda$ vortex moves downstream, the vortex head develops upward along the wall at a certain angle. Owing to the adhesion of the vortex legs to the wall, the vortex head moves faster than the vortex legs, and the $\Lambda$ vortex gradually stretches along the streamwise direction. This increases the vorticity of the $\Lambda$ vortex, inducing a strong upward movement in the fluid contained inside the vortex head and the vortex legs. In contrast, the fluid outside the vortex sweeps down the wall, eventually leading to a burst of turbulence.

The $\mathrm{Q}$ criterion was adopted to identify the threedimensional (3D) vortex structure in the flow field and to model the vortex structure, its evolution, and its interaction. In the region where the second invariant of the velocity gradient tensor is positive $(\mathrm{Q}>0)$, there is a swirling motion in the velocity field and a concentrated area in the vorticity field, which represent the vortex. The $\mathrm{Q}=1.7 \times 10^{6}$ isosurface with the velocity contour was selected to better display the vortex structure. Figure 12 and Fig. 13 present the 3D vortex structures of the turbulence boundary layer for $U=3 \mathrm{~m} / \mathrm{s}$ and $U=5$ $\mathrm{m} / \mathrm{s}$, respectively, with the flow direction from left to right. For $U=3 \mathrm{~m} / \mathrm{s}$, the vortex structure in the boundary layer of the $\mathrm{S}$ structure wall was chaotic, and the $\Lambda$ vortex at the back end of the plane was broken, resulting in turbulence. In contrast, the 


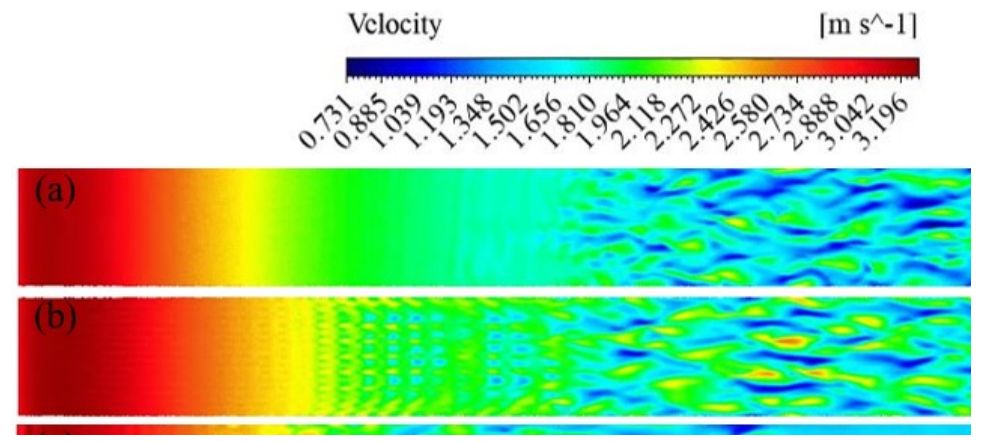

Fig. 10. Streak structures: (a) S-3; (b) G-3; and (c) GM-3.

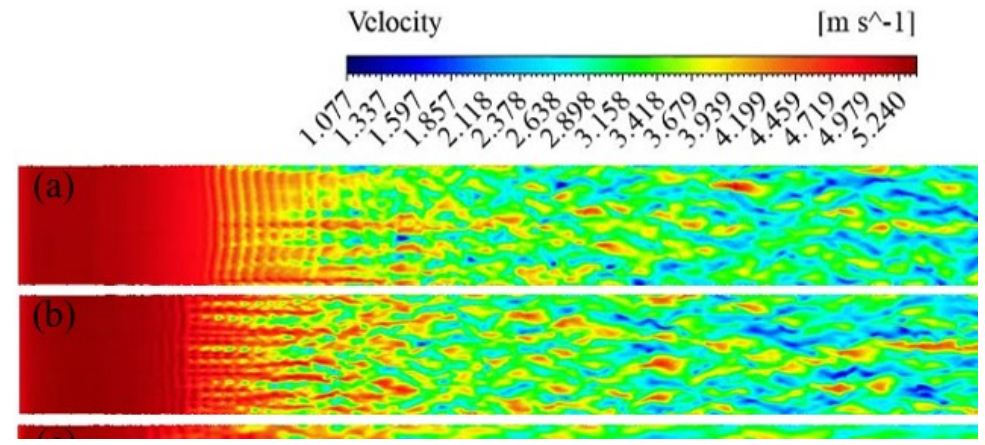

Fig. 11. Streak structures: (a) S-5; (b) G-5; and (c) GM-5.

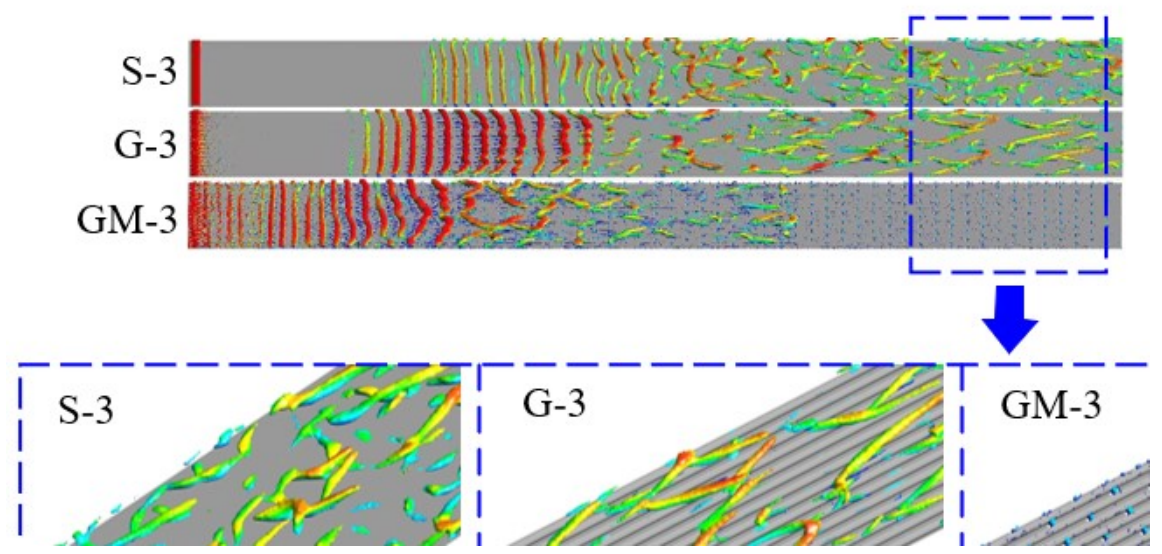

Fig. 12. 3D vortex structure $(U=3 \mathrm{~m} / \mathrm{s})$.

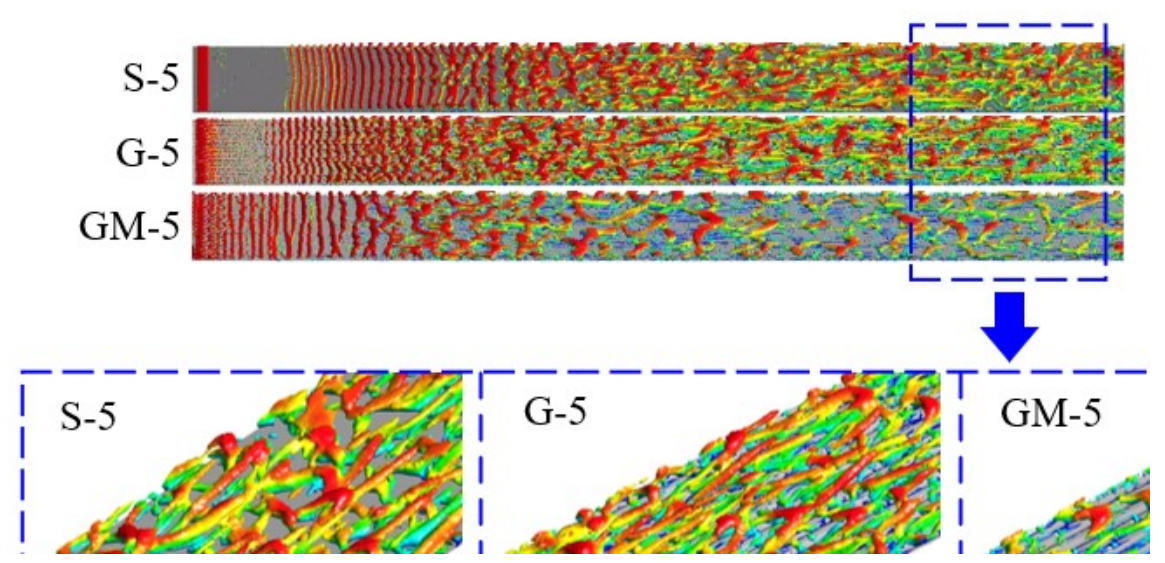

Fig. 13. 3D vortex structure $(U=5 \mathrm{~m} / \mathrm{s})$. 
vortex structure of the G structure was orderly and complete, with a distinct vortex head and vortex legs, and exhibiting an intersection tendency. The vortex tube was elongated along the streamwise direction by the action of the grooves and did not break easily. There was almost no vortex structure at the end of the wall of the GM structure, except for small vortices near the mucus secretion holes. The vortex structure for $U=5 \mathrm{~m} / \mathrm{s}$ was more complicated than that for $U=3 \mathrm{~m} / \mathrm{s}$. However, the control effect of the G and GM structures on the vortex structure remained intact. Therefore, the grooved structure can regulate the shape of the $\Lambda$ vortices, and the mucous membrane can reduce the number of $\Lambda$ vortices. Therefore, the best drag reduction characteristic was obtained with the GM structure wall surface.

\section{Conclusion}

A bionic structure composed of grooves and a mucous membrane (GM) exhibits good turbulent drag reduction performance and is suitable for application in underwater vehicles. Herein, we performed hydrodynamic simulations (LES) to study the frictional drag reduction characteristics in the turbulent boundary layer of a bionic GM structure wall.

(1) Mucus secretion from holes on the wall of the grooved structure can evenly distribute the Carreau viscoelastic fluid on the wall to form a mucous membrane, which plays an important role in frictional drag reduction. The shear-thinning characteristics of the mucous membrane of the Carreau model can suitably control turbulence.

(2) The GM structure has a better drag reduction effect than the G structure. When Re is small, the mucous membrane can increase the $D R$ by approximately $2 \%$. When $\mathrm{Re}$ is high, the drag reduction effect of the mucous membrane becomes stronger, increasing the $D R$ by approximately $8 \%$. In general, the higher the Re, the stronger the drag reduction effect. For a smooth wall, the $D R$ of the bionic GM structure is more than $20 \%$.

(3) Both the G and GM bionic structures can weaken the turbulence peak and shift it away from the wall. This reduces the impact of the turbulence on the wall and the frictional drag. The GM structure has the highest impact on the turbulence statistics.

(4) The bionic structure actively controls the turbulent coherent structure. The streak structure near the wall of the bionic structure reduced, and its shape was regularized. The grooved structure improved the shape of the $\Lambda$ vortex structure, and the mucous film reduced the number of $\Lambda$ vortices. Thus, the bionic GM structure can optimize the coherent structure and significantly reduce frictional drag.

\section{ACKNOWLEDGEMENTS}

This work was supported by the Major Basic Research Projects of Natural Science Foundation of Shandong Province, China (No. ZR2020ZD31).

\section{REFERENCES}

Asrafi, I., M. Yerizam, S. Effendi and A. Mataram (2019). Micro Hydro Electric Power Plant (MHEP) Prototype A Study Of The Effect Of Blade Numbers Toward Turbine Rotational Velocity, Symposium of Emerging Nuclear Technology and Engineering Novelty (Senten 2018) 1198.

Cai, W. H. (2016). Viscoelastic hydrodynamics, Science Press, China.

Cui, J. and Y. B. Fu (2012). A Numerical Study on Pressure Drop in Microchannel Flow with Different Bionic Micro-Grooved Surfaces. Journal of Bionic Engineering 9(1), 99-109.

Dou, Z. L., J. D. Wang and D. R. Chen (2012). Bionic Research on Fish Scales for Drag Reduction. Journal of Bionic Engineering 9(4), 457-464.

Gao, J. Q. (2006). The surface characteristics of carp scales and the lubricating properties of loach body surface mucus. Thesis, Jilin University, China.

Giesekus, H. (1982). A simple constitutive equation for polymer fluids based on the concept of deformation-dependent tensorial mobility. Journal of Non-Newtonian Fluid Mechanics 11(1-2), 69-109.

Graham, M. D. (2014). Drag reduction and the dynamics of turbulence in simple and complex fluids. Physics of Fluids 26(10).

Hefner, J. N., D. M. Bushnell and M. J. Walsh (1983). Research on non-planar wall geometries for turbulence control and skinfriction reduction.

Heidarian, A., H. Ghassemi and P. Liu (2018). Numerical Analysis of the Effects of Riblets on Drag Reduction of a Flat Plate. Journal of Applied Fluid Mechanics 11(3), 679-688.

Jiang, J. B., X. D. Peng, J. Y. Li and Y. Chen (2016). A Comparative Study on the Performance of Typical Types of Bionic Groove Dry Gas Seal based on Bird Wing. Journal of Bionic Engineering 13(2), 324-334.

Ke, G. X., G. Pan, Q. G. Huang, H. B. Hu and Z. Y. Liu (2009). Review of Research on Underwater Drag Reduction Technology. Advances in Mechanics 39(5), 546-554.

Liu, D. M., X. F. Tao, Y. F. Wang, B. Cai, M. Du and Q. Zheng (2010). Rheological Behavior of Loach Surface Mucus. Acta Polymerica Sinica (04), 468-473.

Liu, F., D. J. Liu, W. J. Zhou, S. Wang, F. Chen and J. J. Wei (2020). Weakening or losing of surfactant drag reduction ability: A coarsegrained molecular dynamics study. Chemical Engineering Science 219.

Liu, W. L., H. J. Ni, P. Wang and Y. Zhou (2020). An investigation on the drag reduction 
performance of bioinspired pipeline surfaces with transverse microgrooves. Beilstein Journal of Nanotechnology 11, 24-40.

Liu, B. R., Lin, J. Z., Ku, X. K. and Yu, Z. S. (2021). Elasto-inertial particle migration in a confined simple shear-flow of Giesekus viscoelastic fluids, Particulate Science and Technology 39(6), 726-737.

Majd, A., A. Ahmadi and A. Keramat (2016). Investigation of Non-Newtonian Fluid Effects during Transient Flows in a Pipeline. Strojniski Vestnik-Journal of Mechanical Engineering 62(2), 105-115.

Oldroyd, J. G. (1950). On the Formulation of Rheological Equations of State. Proceedings of the Royal Society of London. Series $A$. Mathematical and Physical Sciences 200(1063), 523-541.

Rajappan, A. and G. H. McKinley (2020). Cooperative drag reduction in turbulent flows using polymer additives and superhydrophobic walls. Physical Review Fluids 5(11).

Seo, E., G. Y. Yoon, H. N. Kim, J. H. Lim, S. Kim, B. Kim, K. H. Kim and S. J. Lee (2020). Morphological features of mucous secretory organ and mucous secretion of loach Misgurnus anguillicaudatus skin for friction drag reduction. Journal of Fish Biology 96(1), 83-91.

Shashank, H. J. and K. R. Sreenivas (2020). Effect of polymer solutions on the propagation and structure of freely translating vortex rings. Chemical Engineering Science 228,115961.

Song, X. W., G. G. Zhang, Y. Wang and S. G. Hu (2011). Use of bionic inspired surfaces for aerodynamic drag reduction on motor vehicle body panels. Journal of Zhejiang UniversityScience A 12(7), 543-551.

Tamano, S., H. Uchikawa, J. Ito and Y. Morinishi (2018). Streamwise variations of turbulence statistics up to maximum drag reduction state in turbulent boundary layer flow due to surfactant injection. Physics of Fluids 30(7).

Tanner, R. I. (1975). Stresses in Dilute Solutions of Bead-Nonlinear-Spring Macromolecules, III. Friction Coefficient Varying with Dumbbell Extension. Journal of Rheology 19, 557-582.

Toms, B. A. (1948). Some observations on the flow of linear Polymer solutions through straight tubes at large Reynolds numbers. First International Congress on Rheology, Amsterdam, Netherlands.

Vilalta, G., M. Silva and A. Blanco (2016). Influence Study of the Viscoelastic Fluids
Features in Drag Reduction in Laminar Regime Flow in Pipeline. 3rd International Conference on Manufacturing and Industrial Technologies, Istanbul, Turkey.

Wakimoto, T., K. Araga and K. Katoh (2018). Simultaneous determination of micellar structure and drag reduction in a surfactant solution flow using the fluorescence probe method. Physics of Fluids 30(3).

Walsh, M. J. (1983). Riblets as a Viscous Drag Reduction Technique. Aiaa Journal 21(4) 485486.

Walsh, M. J. (1990). Viscous Drag Reduction in Boundary Layers. Progress in Astronautics Aeronautics 123, 203-216.

Xi, L. (2019). Turbulent drag reduction by polymer additives: Fundamentals and recent advances. Physics of Fluids 31(12).

$\mathrm{Xu}$, C. (2018). Measurement of rheological properties of puffer surface mucus and research on the application of bionic drag reduction. Thesis, Jiangsu University of Science and Technology, China.

Yanuar, Gunawan, K. T. Waskito and S. Ogata (2017). Effect of Agar Jelly Coating in Rectangular Pipe to Flow Drag Reduction. Journal of Applied Fluid Mechanics 10(4), 1161-1166.

Zakin, J. L. and H. W. Bewersdorff (1998). Surfactant Drag Reduction. Reviews in Chemical Engineering 14(5), 253-320.

Zhang, K., C. Ma, B. Zhang, B. Zhao and Q. Wang (2020a). Numerical Simulation Study on Bionic Mucus Drag Reduction of Underwater Vehicle. International Journal of Fluid Mechanics Research 47(4), 371-385.

Zhang, M. M., Z. L. Zhang and M. Zhao (2020b). Investigation on the Mechanism of Drag Modification over Triangular Riblets. Journal of Applied Fluid Mechanics 13(4), 1093-1106.

Zhang, X., X. L. Duan and Y. Muzychka (2020c). Drag reduction by linear flexible polymers and its degradation in turbulent flow: A phenomenological explanation from chemical thermodynamics and kinetics. Physics of Fluids 32(1), 013101-.

Zhang, Z. S. (2017). Turbulence Theory and Simulation (Second Edition), Tsinghua University Press.

Zhu, L. and L. Xi (2019). Vortex dynamics in lowand high-extent polymer drag reduction regimes revealed by vortex tracking and conformation analysis. Physics of Fluids 31(9). 